Article

\title{
Scattering as a Quantum Metrology Problem: A Quantum Walk Approach
}

\author{
Francesco Zatelli ${ }^{1}$, Claudia Benedetti ${ }^{1, *}$ (i) and Matteo G. A. Paris ${ }^{1,2}$ (i) \\ 1 Dipartimento di Fisica 'Aldo Pontremoli', Università degli Studi di Milano, I-20133 Milano, Italy; \\ francesco.zatelli@gmail.com (F.Z.); matteo.paris@fisica.unimi.it (M.G.A.P.) \\ 2 INFN, Sezione di Milano, I-20133 Milano, Italy \\ * Correspondence: claudia.benedetti@unimi.it
}

Received: 14 October 2020; Accepted: 17 November 2020; Published: 19 November 2020

check for updates

\begin{abstract}
We address the scattering of a quantum particle by a one-dimensional barrier potential over a set of discrete positions. We formalize the problem as a continuous-time quantum walk on a lattice with an impurity and use the quantum Fisher information as a means to quantify the maximal possible accuracy in the estimation of the height of the barrier. We introduce suitable initial states of the walker and derive the reflection and transmission probabilities of the scattered state. We show that while the quantum Fisher information is affected by the width and central momentum of the initial wave packet, this dependency is weaker for the quantum signal-to-noise ratio. We also show that a dichotomic position measurement provides a nearly optimal detection scheme.
\end{abstract}

Keywords: quantum walks; scattering; quantum metrology; quantum Fisher information; optimal measurement

\section{Introduction}

Since the Rutherford experiment [1], scattering has played a central role in the study of unknown interactions in many fields of physics [2-4]. At its core, a scattering experiment may be viewed as a parameter-estimation problem. Indeed, the scattering potential can be modeled with a set of unknown parameters that characterize the evolution of the quantum particles that impinge on it. Estimating the value of those parameters then involves measurements that are performed on the scattered state, followed by a collection of outputs that are used to build estimators for the parameters. If we consider scattering as an estimation problem, we can study the maximum amount of information that can be extracted from a single measurement on the quantum system, and we can assess the performance of feasible detection schemes. All these questions find answers in the theory of local quantum estimation, which has the aim of quantifying the best precision of an estimation procedure [5]. Indeed, in the past few years, local quantum estimation theory has been applied to a variety of problems, such as the estimation of the relevant parameters of quantum structured baths [6-10], graph and lattice properties [11-13], and classical processes [14].

In this work, we analyze the one-dimensional scattering of a quantum particle from a potential barrier with the aim of inferring its height. The particle moves on a set of discrete positions, and it is thus described as a continuous-time quantum walk $(\mathrm{CT} \mathrm{QW})$ on the line with a central barrier. The barrier is implemented by a detuning of the energy of the central site with respect to the other sites. As a matter of fact, the analysis of the evolution of a quantum walk in the presence of a barrier is strongly connected with the study of defects and impurities in implementations of QW [15-18]. A detuning in the on-site energy 
of a site can be interpreted as a defect that influences the dynamics and the scattering properties of the walker. Understanding the role of imperfections is of fundamental importance for a realistic description of the QWs. In fact, knowing how a protocol or an algorithm [19-23] is affected by impurities and noise allows us to hinder or even neutralize detrimental effects.

Inspired by previous works on the discretization of continuous-systems [24,25], we first derive scattered states on the infinite line of discrete positions. In order to consider physically relevant states for the walker, we initialize the particle in a Gaussian wave packet with central initial momentum $k_{0}$ and standard deviation $\sigma$. We evaluate the transmission probability through the barrier and the maximum extractable information as a function of these two free parameters. We show that the quantum Fisher information (QFI) is strongly affected by the value of the initial central momentum of the walker, but only slightly by the initial spread of the wave packet. The quantum signal-to-noise ratio has a maximum corresponding to the optimal value of the barrier height that can be better estimated. Finally, we consider a feasible measurement, i.e., a dichotomic position measurement, and we compare its Fisher information (FI) with the QFI. We show that this measurement is nearly optimal, i.e., its FI is close to the QFI in almost all the parameter space we consider.

The paper is organized as follows: In Section 2, we introduce the concept of CTQW with inhomogeneous on-site energies, and in Section 3, we briefly review the main concepts of local quantum estimation theory. In Section 4, we introduce the free-particle scattering states, and then, we use them to build the physically relevant wave packets, whose transmission and reflection probabilities are derived. In Section 5, we compute the QFI for initial Gaussian wave packets, and we compare its value with the FI of a dichotomic position measurement. Finally, in Section 6, we draw our conclusions.

\section{Quantum Walks with Inhomogeneous On-Site Energies}

A CTQW model describes the evolution of a quantum particle over a discrete set of positions, continuously in time [26,27]. It evolves in an $\mathrm{N}$-dimensional Hilbert space with orthonormal basis states

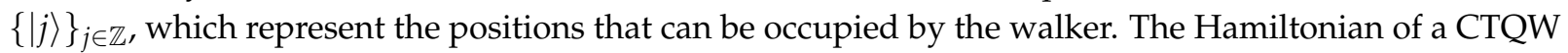
on the line with inhomogeneous on-site energies $\epsilon_{j}$ and uniform couplings $J_{0}$ has the expression $(\hbar=1)$ :

$$
H=\sum_{j} \epsilon_{j}|j\rangle\langle j|-J_{0} \sum_{j}(|j\rangle\langle j+1|+| j+1\rangle\langle j|) .
$$

Without loss of generality, we fix $J_{0}=1$, thus expressing time and $\epsilon_{j}$ in units of $J_{0}$. If we set $\epsilon_{j}=2 \forall j$, we recover the graph Laplacian $L$, i.e., $H=-L$. It is worth mentioning that for the one-dimensional lattice, $L$ represents the discretized version of Laplace operator $\nabla^{2}$, and $-L$ is kinetic energy operator of a particle with mass $m=\frac{1}{2}$ constrained to a discrete set of positions [28].

Given a set of on-site energies $\left\{\epsilon_{j}\right\}$, it is possible to separate the Hamiltonian into a kinetic and a potential operator, $L$ and $V$ respectively. The Hamiltonian can thus be written as $H=-L+V$ with:

$$
L=\sum_{j}[-2|j\rangle\langle j|+| j\rangle\langle j+1|+| j+1\rangle\langle j|] \text { and } V=\sum_{j} V_{j}|j\rangle\left\langle j\left|=\sum_{j}\left(\epsilon_{j}-2\right)\right| j\right\rangle\langle j|
$$

highlighting the fact that for $\epsilon_{j}=2 \forall j$, the unperturbed Laplacian Hamiltonian is obtained. Due to the tridiagonal form of the matrix $H$, the eigenvalue equation $H\left|\psi^{(k)}\right\rangle=E_{k}\left|\psi^{(k)}\right\rangle$ can be recast in the form of a three-term recurrence relation. By explicitly writing $H$ in terms of the Laplacian and potential parts and projecting into a basis state $|j\rangle$, we obtain $\left\langle j|-L+V| \psi^{(k)}\right\rangle=E_{k}\left\langle j \mid \psi^{(k)}\right\rangle$ and the recurrence relation:

$$
-\psi_{j+1}^{(k)}+2 \psi_{j}^{(k)}-\psi_{j-1}^{(k)}+V_{j} \psi_{j}^{(k)}=E_{k} \psi_{j}^{(k)},
$$


where $\left|\psi^{(k)}\right\rangle=\sum_{j} \psi_{j}^{(k)}|j\rangle$. Equation (3) is easily identifiable with the discretization in the position basis of the time-independent Schrödinger equation for a particle of mass $m=\frac{1}{2}$.

In analogy with the continuous case, we introduce the momentum states as the Fourier series of the countable orthonormal set of position eigenstates. In particular, we define the momentum state $|k\rangle$ through a discrete-time Fourier transform (DTFT):

$$
\begin{aligned}
& |k\rangle=\frac{1}{\sqrt{2 \pi}} \sum_{j \in \mathbb{Z}} e^{i k j}|j\rangle, \quad k \in(-\pi, \pi] \\
& |j\rangle=\frac{1}{\sqrt{2 \pi}} \int_{-\pi}^{\pi} e^{-i k j}|k\rangle \mathrm{d} k, \quad j \in \mathbb{Z} .
\end{aligned}
$$

If no external potential is considered, i.e., $V_{j}=0 \forall j$, the states $\{|k\rangle\}$ are solutions to Equation (3) with $\psi_{j}^{(k)}=e^{i k j}$ and corresponding energies $E_{k}=2-2 \cos (k)$. The dispersion relation implies that the phase velocity $v_{p}$ and the group velocity $v_{g}$ are:

$$
v_{p}=\frac{E_{k}}{k}=\frac{2-2 \cos (k)}{k}, \quad v_{g}=\frac{\partial E_{k}}{\partial k}=2 \sin (k) .
$$

Thus, the momentum states (4) are the discretization of the plane waves with the dispersion relation typical of the tight-binding models [29]. We identify these states as free particle states because, in analogy with the continuous case, plane waves are the eigenstates of a purely kinetic Hamiltonian. This suggests that the separation of the QW Hamiltonian into a kinetic term and a potential one is indeed meaningful. In the following, we are going to introduce an obstacle, i.e., an external potential that causes an inhomogeneity on the on-site energies.

\section{Tools of Local Quantum Estimation Theory}

Before analyzing the QW scattering from a barrier, we review a few key concepts in the theory of local quantum estimation. Consider a sample of $M$ independent outcomes of a measurement $\left\{x_{1}, x_{2}, \ldots, x_{M}\right\}$ drawn from the probability distribution $p(x \mid \Delta)$, where $\Delta$ is an unknown parameter we wish to estimate. The Cramèr-Rao (CR) inequality imposes a lower bound on the variance of any unbiased estimator $\hat{\Delta}\left(\left\{x_{1}, x_{2}, \ldots, x_{M}\right\}\right)$ for such a parameter:

$$
\operatorname{Var}(\hat{\Delta}) \geq \frac{1}{M F(\Delta)}
$$

where $F(\Delta)$ is the Fisher information, defined as:

$$
F(\Delta)=\int\left(\frac{\partial \ln p(x \mid \Delta)}{\partial \Delta}\right)^{2} p(x \mid \Delta) \mathrm{d} x=\int\left(\frac{\partial p(x \mid \Delta)}{\partial \Delta}\right)^{2} \frac{1}{p(x \mid \Delta)} \mathrm{d} x
$$

The quantum version of the CR bound is derived by generalizing the concept of FI. This is done by maximizing the FI over all possible measurements, and the obtained quantity is called quantum Fisher information $H(\Delta)$. A detailed derivation of the QFI can be found in [30]. The quantum CR bound takes the following form:

$$
\operatorname{Var}(\hat{\Delta}) \geq \frac{1}{M H(\Delta)} .
$$

and follows from the inequality $F(\Delta) \leq H(\Delta)$, which provides the basis for the identification of the QFI with the ultimate bound to precision of any unbiased estimator. The aim of local quantum estimation 
theory is to determine the maximum extractable information from a quantum probe, whose state depends on the value of the parameter. If only pure states are considered as probes, i.e., a parameter-dependent family of quantum states $\left|\psi_{\Delta}\right\rangle$, the QFI can be explicitly written as [30]:

$$
H(\Delta)=4\left[\left\langle\partial_{\Delta} \psi_{\Delta} \mid \partial_{\Delta} \psi_{\Delta}\right\rangle-\left|\left\langle\psi_{\Delta} \mid \partial_{\Delta} \psi_{\Delta}\right\rangle\right|^{2}\right],
$$

where $\left|\partial_{\Delta} \psi_{\Delta}\right\rangle$ represents the derivative of the state with respect to the parameter $\Delta$. A suitable figure of merit that can be used in order to evaluate the estimability of a parameter is the quantum signal-to-noise ratio (QSNR):

$$
R(\Delta)=\Delta^{2} H(\Delta),
$$

which provides an upper bound to the signal-to-noise ratio $\hat{\Delta}^{2} / \operatorname{Var}(\hat{\Delta})$ of any detection scheme.

\section{Scattering in the Presence of an Obstacle}

Let us now consider a situation where there is an obstacle placed in the middle of the chain. The obstacle, or barrier, has the width of a single site, i.e., all sites have the same energy $\epsilon_{j}=2$, except for the central one $|0\rangle$, which has a detuning $\Delta$, such that $\epsilon_{0}=2+\Delta$. Thus, the Hamiltonian defined in Equation (1) is modified by placing the obstacle at $j=0$, and it becomes:

$$
H=\sum_{j \in \mathbb{Z}}(2|j\rangle\langle j|-| j+1\rangle\langle j|-| j\rangle\langle j+1|)+\Delta|0\rangle\langle 0| .
$$

The site $j=0$ has on-site energy $\epsilon_{0}=2+\Delta$ or, alternatively said, potential $V_{0}=\Delta$. In order to study the scattering properties of such model, we start by deriving the scattering states.

\subsection{Scattering States}

Scattering states for one-dimensional systems in the continuous-space case are known for a variety of potentials [31]. We now want to derive such states for the discrete system under consideration. The generic stationary scattering state $\left|\psi_{s}\right\rangle$ with fixed momentum $k$ can be written as a linear combination of free particle states, namely:

$$
\left\langle j \mid \psi_{s}\right\rangle=\left\{\begin{array}{ll}
A e^{i k j}+B e^{-i k j}, & j \leq 0 \\
C e^{i k j}, & j \geq 0
\end{array},\right.
$$

where the terms proportional to $A, B$, and $C$ correspond to the incident, the reflected, and the transmitted wave, respectively. The coefficients are calculated imposing that the two parts of the state (before and after the obstacle) are properly connected at $j=0$, i.e., by discretizing the continuity conditions, and using the recurrence relations (3), i.e., $\left\langle-1 \mid \psi_{s}\right\rangle-\Delta\left\langle 0 \mid \psi_{s}\right\rangle+\left\langle 1 \mid \psi_{s}\right\rangle=2 \cos (k)\left\langle 0 \mid \psi_{s}\right\rangle$, which represent the discontinuity introduced by the obstacle. Therefore, the reflection $R=\frac{|B|^{2}}{|A|^{2}}$ and transmission $T=\frac{|C|^{2}}{|A|^{2}}$ coefficients can be easily calculated through:

$$
\left\{\begin{array} { l } 
{ A + B = C } \\
{ A e ^ { - i k } + B e ^ { i k } = C ( 2 \operatorname { c o s } ( k ) + \Delta - e ^ { i k } ) }
\end{array} \quad \left\{\begin{array}{l}
B=\frac{1}{\frac{2 i \sin k}{\Delta}-1} A \\
C=\frac{1}{1-\frac{\Delta}{2 i \sin k}} A
\end{array},\right.\right.
$$

and they have the expressions:

$$
R(\Delta, k)=\frac{1}{1+\frac{4 \sin ^{2}(k)}{\Delta^{2}}}, \quad T(\Delta, k)=\frac{1}{1+\frac{\Delta^{2}}{4 \sin ^{2}(k)}} .
$$


These coefficients closely resemble those corresponding to a delta potential in a continuous system [31]; in particular, the coefficients only depend on $\Delta^{2}$, meaning that there is no difference between an attractive or repulsive potential as concerns scattering. If $\Delta$ is fixed, $T$ is maximum for $k=\frac{\pi}{2}$, which corresponds to the highest group velocity (but not to the highest energy). Consistently, at the same value of $k, R$ has a minimum. As the absolute value of $\Delta$ is increased, the transmission coefficient drops to smaller values, as reported in Figure 1. For every incident $|k\rangle$, we may thus define:

$$
S|k\rangle=\frac{B}{A}|-k\rangle+\frac{C}{A}|k\rangle
$$

where we introduced a scattering matrix $S$ whose elements give information on the reflection and transmission coefficients [31]. If we set $A=|A|$ and we highlight the phases of the reflected and transmitted waves, we obtain:

$$
S|k\rangle=\frac{|B|}{|A|} e^{i \phi_{B}}|-k\rangle+\frac{|C|}{|A|} e^{i \phi_{C}}|k\rangle=e^{i \phi_{B}}\left(\sqrt{R(\Delta, k)}|-k\rangle+\sqrt{T(\Delta, k)} e^{i\left(\phi_{C}-\phi_{B}\right)}|k\rangle\right) .
$$

The relative phase $e^{i\left(\phi_{C}-\phi_{B}\right)}$ can be computed from the ratio $\frac{C}{B}$ from Equation (14) and is equal to $\pi / 2$. It follows that:

$$
S|k\rangle=e^{i \phi_{B}(\Delta, k)}(\sqrt{R(\Delta, k)}|-k\rangle+i \sqrt{T(\Delta, k)}|k\rangle),
$$

with the phase $\phi_{B}(\Delta, k)=\arctan \left(\frac{2 \sin (k)}{\Delta}\right)$.
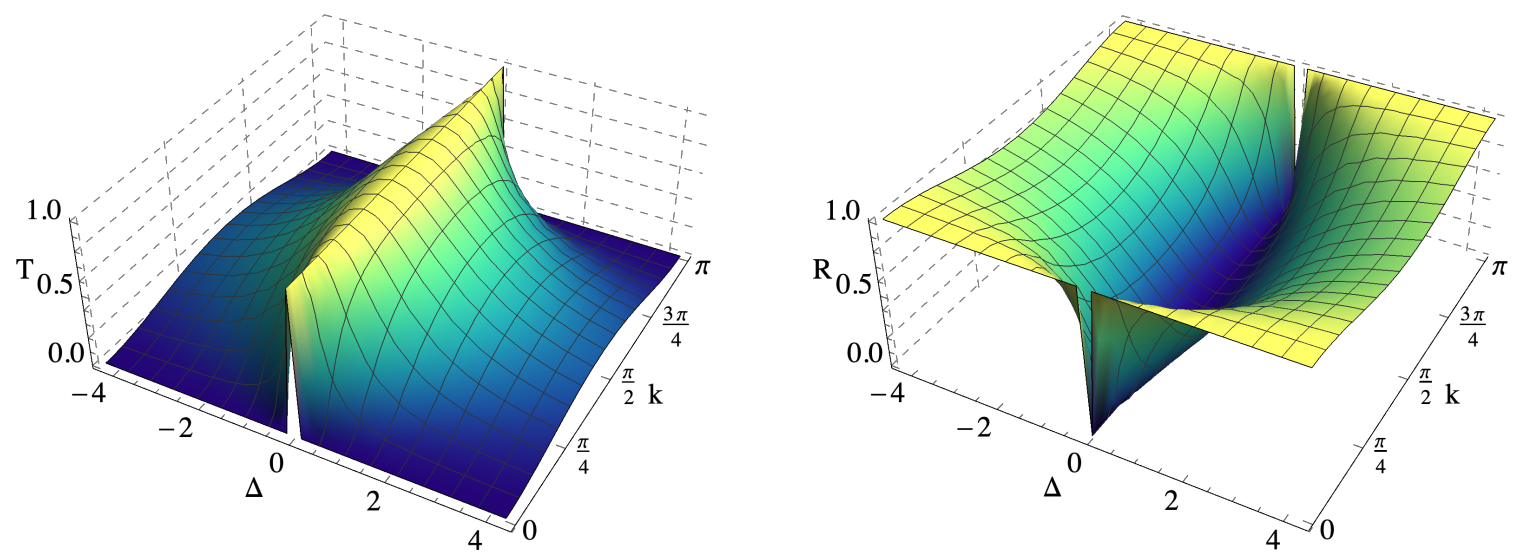

Figure 1. Transmission and reflection coefficients $T$ and $R$ as a function of $\Delta$ and $k$.

It is possible to define the reflection and transmission coefficients for more general states. Given an initial localized wave packed $\left|\psi_{0}\right\rangle$ placed on the left of the obstacle, its time-evolved state is:

$$
|\psi(t)\rangle=e^{-i H t}\left|\psi_{0}\right\rangle
$$

We define the time-dependent probabilities:

$$
\rho(t)=\sum_{j<0}|\langle j \mid \psi(t)\rangle|^{2}, \quad \tau(t)=\sum_{j>0}|\langle j \mid \psi(t)\rangle|^{2}, \quad \delta(t)=|\langle 0 \mid \psi(t)\rangle|^{2} .
$$


The quantities $\rho(t)$ and $\tau(t)$ are indeed the probability of finding the walker before and after the obstacle, respectively. The defect coefficient $\delta(t)$ is the remaining probability, namely the probability of finding the particle on the obstacle site. In particular, when the scattering is over, the coefficient $\delta(t)$ is expected to vanish, and consequently, $\rho(t)+\tau(t)=1$.

\subsection{Gaussian Wave Packets}

The vector described by Equation (18) is the mathematical building block from which we derive the asymptotic values of the quantities of interest; however, it is not normalizable and does not represent a physical state. For this reason, we now introduce more realistic states that are spatially localized. In particular, we consider a discretized version of a Gaussian wave packet:

$$
\left|\mathcal{G}_{k_{0}}\right\rangle=\mathcal{N} \sum_{j \in \mathbb{Z}} e^{-\frac{(j-\mu)^{2}}{2 \sigma^{2}}} e^{i k_{0} j}|j\rangle .
$$

The probability distribution of this state is a discretized Gaussian function with mean $\mu$ and variance $\frac{\sigma^{2}}{2}$. $\mathcal{N}$ is a normalization constant, while the parameter $k_{0} \in(-\pi, \pi]$ represents the mean of the probability distribution in the momentum basis. The $\left|\mathcal{G}_{k_{0}}\right\rangle$ state in the momentum basis is still Gaussian under proper assumptions, and it has the expression:

$$
\begin{aligned}
\left|\mathcal{G}_{k_{0}}\right\rangle & =\int_{-\pi}^{\pi} g_{k_{0}}(k)|k\rangle d k, \\
\text { with } \quad g_{k_{0}}(k) & =\left\langle k \mid \mathcal{G}_{k_{0}}\right\rangle \approx \sqrt{\frac{\sigma}{\pi^{1 / 2}}} e^{-\frac{\left(k-k_{0}\right)^{2} \sigma^{2}}{2}} e^{-i \mu k} .
\end{aligned}
$$

The detailed derivation of Expression (23) is shown in Appendix A. The crucial approximation made to obtain this expression is to consider narrow wave packets in the reciprocal space (i.e., $\min \left(\left|k_{0}+\pi\right|\right.$, $\left.\left|\pi-k_{0}\right|\right) \gg 1 / \sigma$; see Appendix A). Therefore, the Fourier transform of the Gaussian wave packet is not exactly a Gaussian in the momentum basis. Nevertheless, if the transformed state is sufficiently localized in reciprocal space, Equation (23) is a reasonable approximation.

\subsection{Scattering with Gaussian Wave Packets}

Here, we want to analyze the asymptotic scattering properties of an incident Gaussian wave packet. In order to do so, we exploit the results obtained for single momentum states $|k\rangle$. The Gaussian state in the momentum basis has the expression (22) where the Gaussian weights are included in $g_{k_{0}}(k)$.

We consider a wave packet incident on the obstacle from the left $(j<0)$. Using (18) and linearity, the scattered Gaussian state can be written in the asymptotic limit as:

$$
\begin{aligned}
\left|\psi_{k_{0}, \Delta}\right\rangle & =S\left|\mathcal{G}_{k_{0}}\right\rangle=\int_{-\pi}^{\pi} g_{k_{0}}(k) S|k\rangle d k \\
& =\int_{-\pi}^{\pi} g_{k_{0}}(k) e^{i \phi_{B}(\Delta, k)}(\sqrt{R(\Delta, k)}|-k\rangle+i \sqrt{T(\Delta, k)}|k\rangle) d k \\
& =\int_{-\pi}^{\pi}\left(e^{-i \phi_{B}(\Delta, k)} \sqrt{R(\Delta, k)}\left|g_{-k_{0}}(k)\right| e^{i \mu k}+e^{i \phi_{B}(\Delta, k)} i \sqrt{T(\Delta, k)}\left|g_{k_{0}}(k)\right| e^{-i \mu k}\right)|k\rangle d k,
\end{aligned}
$$

where, in the last line, we used the equalities $\left|g_{k_{0}}(-k)\right|=\left|g_{-k_{0}}(k)\right|, R(\Delta, k)=R(\Delta,-k)$, and $\phi_{B}(\Delta, k)=$ $-\phi_{B}(\Delta,-k)$. By inspection of Equation (24), we learn that the original Gaussian wave packet is divided into the superposition of two wave packets centered around opposite values of momentum $k_{0}$ and $-k_{0}$, corresponding to the transmitted and reflected wave function, respectively. These two wave packets 
are not Gaussian anymore, since they are weighted with scattering coefficients that depend on $k$. It is important to highlight that this description fails if the two wave packets overlap, which can happen if the original state is spread in $k$-space or if its mean is $k_{0} \approx 0$ (or any multiple of $\pi$ ). The assumption of a narrow initial wave packet in $k$-space was already imposed in order to derive Equation (23), while asking for a $k_{0} \neq 0$ corresponds to considering a wave packet with the group velocity different from zero. With these assumptions, the transmission and reflection coefficients can be calculated considering the probabilities of the reflected and transmitted wave packets:

$$
\rho_{\mathcal{G}}\left(k_{0}, \Delta\right)=\int_{-\pi}^{\pi} R(\Delta, k)\left|g_{k_{0}}(k)\right|^{2} d k, \quad \tau_{\mathcal{G}}\left(k_{0}, \Delta\right)=\int_{-\pi}^{\pi} T(\Delta, k)\left|g_{k_{0}}(k)\right|^{2} d k .
$$

This results are confirmed by numerical evaluation of the $\rho(t)$ and $\tau(t)$ coefficients in Equation (20) and shown in Figure 2. The dynamics of the walker is computed through Equation (19) for fixed values of $k_{0}$ and $\Delta$. The figure shows that at long times, i.e., in the asymptotic limit, the transmission probability achieves exactly $\tau_{\mathcal{G}}\left(k_{0}, \Delta\right)$. A large transmission probability is associated with high values of $k_{0}$ and small values of $\Delta$, while a small initial central momentum and a large barrier prevent good transmission.
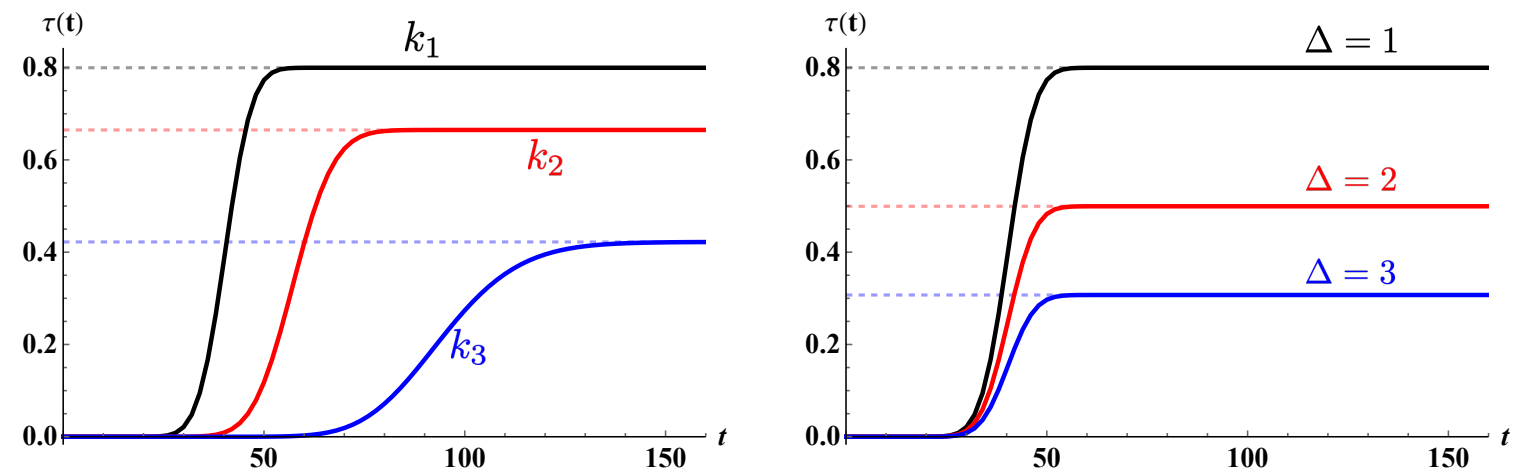

Figure 2. Transmission probability $\tau(t)$. The left plot is for a fixed value of $\Delta=1$ and for decreasing values of $k_{0}=k_{1}, k_{2}, k_{3}$, with $k_{1}=\pi / 2$ (black), $k_{2}=\pi / 4$ (red), $k_{3}=\pi / 7$ (blue). In the right plot, $k_{0}=\pi / 2$ is kept fixed while varying the disorder $\Delta=1$ (black), $\Delta=2$ (red), $\Delta=3$ (blue). The dashed lines correspond to the value of the transmission coefficient $\tau_{\mathcal{G}}\left(k_{0}, \Delta\right)$ in Equation (25). In both plots, we considered $\sigma=15$.

\section{Quantum Estimation of a Scattering Potential}

After having derived the scattered expression of a Gaussian wave packet, we turn our attention to the optimal estimation of the barrier height, i.e., of the parameter $\Delta$. In order to do so, we prepare an initial Gaussian wave packet with initial central momentum $k_{0}$. In a scattering experiment, measurements can be performed only on the scattered state, which has the expression of Equation (24), which we report here for convenience:

$$
\left|\psi_{k_{0}, \Delta}\right\rangle=\int_{-\pi}^{\pi} g_{k_{0}}(k) e^{i \phi_{B}(\Delta, k)}(\sqrt{R(\Delta, k)}|-k\rangle+i \sqrt{T(\Delta, k)}|k\rangle) d k
$$

In order to compute the QFI, Equation (10), we need the derivative:

$$
\begin{aligned}
& \left|\partial_{\Delta} \psi_{k_{0}, \Delta}\right\rangle=\int_{-\pi}^{\pi} g_{k_{0}}(k) e^{i \phi_{B}(\Delta, k)} \times \\
& \quad \times\left[i \partial_{\Delta} \phi_{B}(\Delta, k)(\sqrt{R(\Delta, k)}|-k\rangle+i \sqrt{T(\Delta, k)}|k\rangle)+\left(\frac{\partial_{\Delta} R(\Delta, k)}{2 \sqrt{R(\Delta, k)}}|-k\rangle+i \frac{\partial_{\Delta} T(\Delta, k)}{2 \sqrt{T(\Delta, k)}}|k\rangle\right)\right] d k,
\end{aligned}
$$


and the inner products:

$$
\begin{aligned}
\left\langle\partial_{\Delta} \psi_{k_{0}, \Delta} \mid \partial_{\Delta} \psi_{k_{0}, \Delta}\right\rangle & =\int_{-\pi}^{\pi}\left|g_{k_{0}}(k)\right|^{2}\left(\left[\partial_{\Delta} \phi_{B}(\Delta, k)\right]^{2}+\frac{\left[\partial_{\Delta} R(\Delta, k)\right]^{2}}{4 R(\Delta, k)}+\frac{\left[\partial_{\Delta} T(\Delta, k)\right]^{2}}{4 T(\Delta, k)}\right) d k \\
\left\langle\psi_{k_{0}, \Delta} \mid \partial_{\Delta} \psi_{k_{0}, \Delta}\right\rangle & =i \int_{-\pi}^{\pi}\left|g_{k_{0}}(k)\right|^{2} \partial_{\Delta} \phi_{B}(\Delta, k) d k
\end{aligned}
$$

with $\partial_{\Delta} R(\Delta, k)+\partial_{\Delta} T(\Delta, k)=0$. We remind the reader that in this work, we always assume that the reflected and transmitted wave packets of the post-scattering state do not overlap, neither in position nor in momentum space. Notice that with this assumption, we also exclude slow states, i.e., those states with $k_{0} \approx 0$ or $k_{0} \approx \pi$. The QFI for an initial Gaussian wave packet may be computed through Equation (10):

$$
\begin{aligned}
H_{\mathcal{G}}\left(k_{0}, \Delta\right)= & \int_{-\pi}^{\pi}\left|g_{k_{0}}(k)\right|^{2}\left(\frac{\left[\partial_{\Delta} R(\Delta, k)\right]^{2}}{R(\Delta, k)}+\frac{\left[\partial_{\Delta} T(\Delta, k)\right]^{2}}{T(\Delta, k)}+4\left[\partial_{\Delta} \phi_{B}(\Delta, k)\right]^{2}\right) d k \\
& -4\left(\int_{-\pi}^{\pi}\left|g_{k_{0}}(k)\right|^{2} \partial_{\Delta} \phi_{B}(\Delta, k) d k\right)^{2} \\
= & \frac{16 \sin ^{2} k_{0}}{\left[2+\Delta^{2}-2 \cos \left(2 k_{0}\right)\right]^{2}}+\frac{g_{H}\left(k_{0}, \Delta\right)}{\sigma^{2}}+O\left(1 / \sigma^{3}\right),
\end{aligned}
$$

where the explicit expression of $g_{H}\left(k_{0}, \Delta\right)$ is reported in Appendix B.

The typical behavior of the QFI as a function of $\Delta$ and the initial central momentum $k_{0}$ is shown in Figure 3. Since we want to avoid overlaps of the reflected and transmitted wave functions in momentum space, we exclude values for $k_{0}$ in the neighborhood of $k_{0}=0$ and $k_{0}=\pi$. The QFI is symmetric under the exchange of the sign of the barrier, i.e., $\Delta \rightarrow-\Delta$, and it has a maximum centered in $\Delta=0$. Small values of the barrier height $|\Delta| \ll 1$ have a larger QFI with respect to higher barriers. The spread of the wave packet $\sigma$ affects the maximum precision only for $|\Delta| \ll 1$, as shown in the upper panel of Figure 4 . From these plots, we can also see that the initial central momentum has an important role: in fact, as $k_{0}$ is increased from small values to $\frac{\pi}{2}$, the maximum of the QFI decreases.

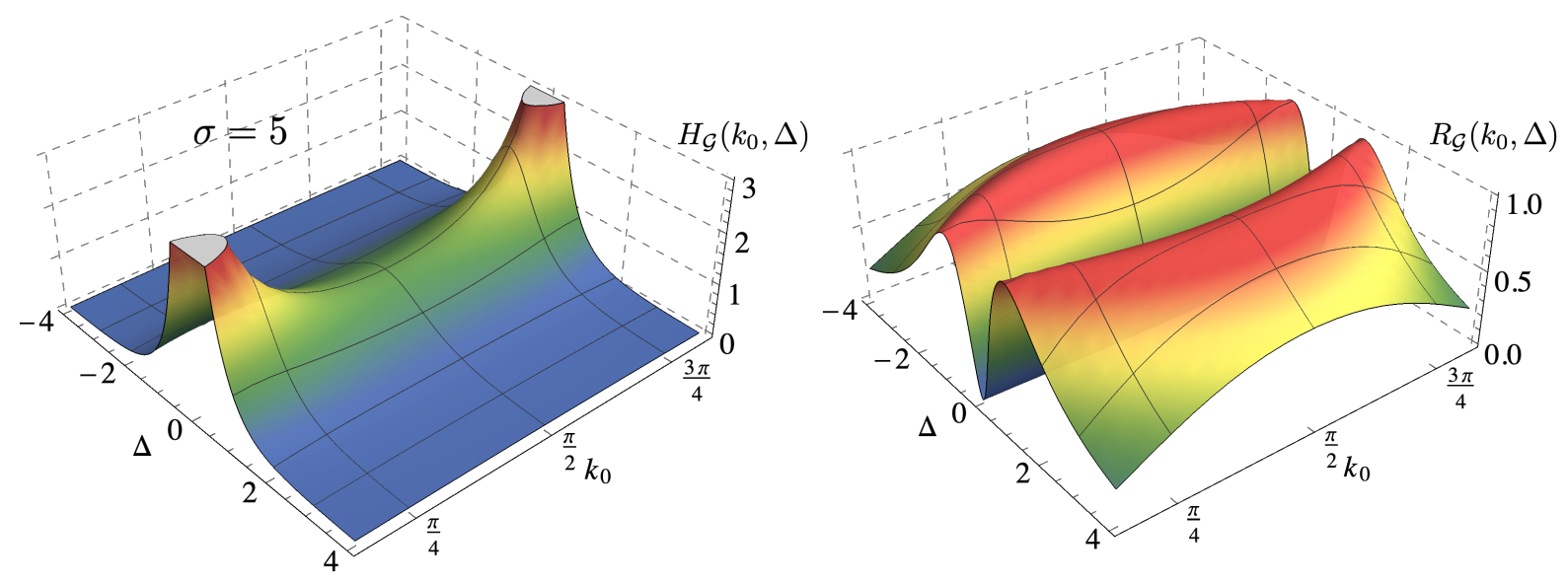

Figure 3. Left: QFI $H_{\mathcal{G}}\left(k_{0}, \Delta\right)$ for an initial Gaussian wave packet with $\sigma=5$. Right: QSNR $R_{\mathcal{G}}\left(k_{0}, \Delta\right)$ for the same initial Gaussian wave packet. 

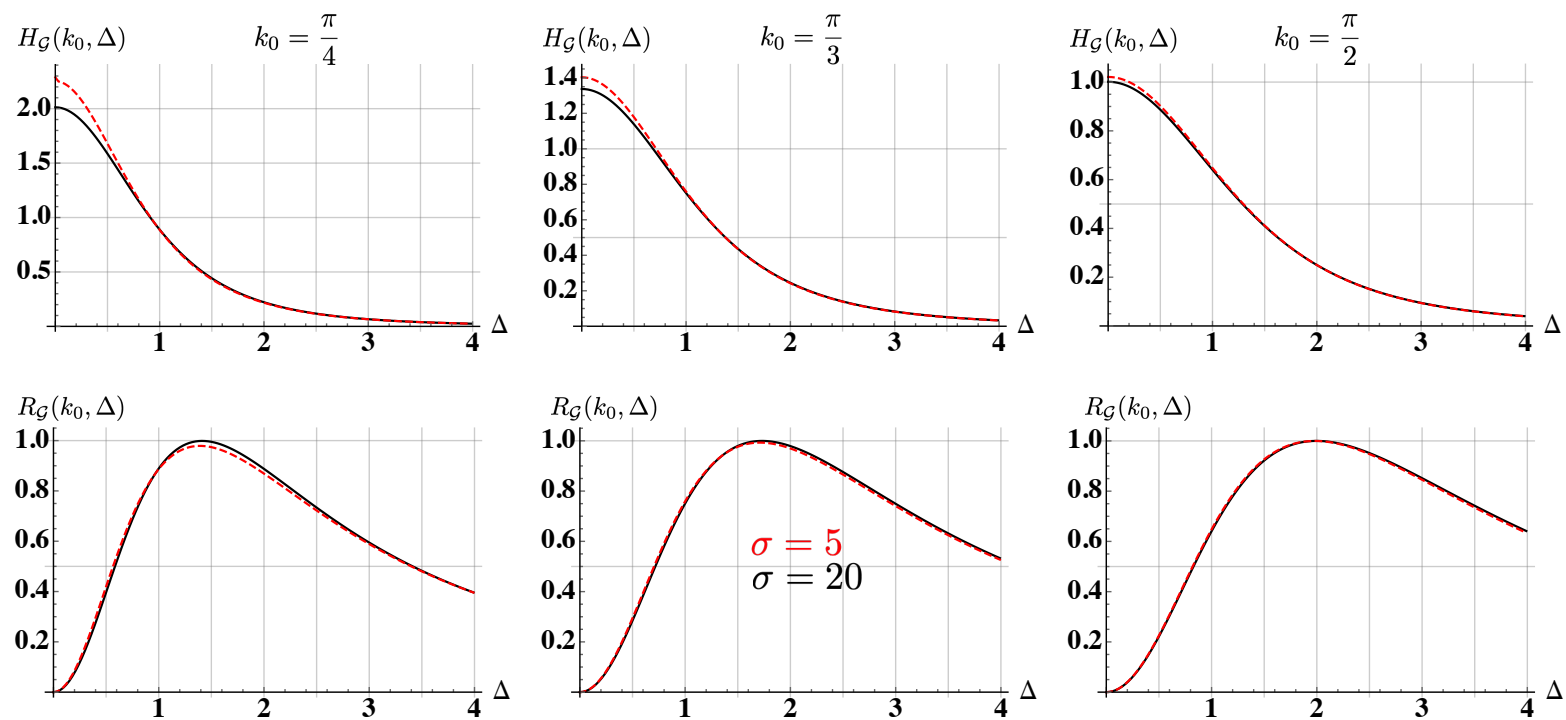

Figure 4. Comparison between the QFI (upper panel) and the QSNR (lower panel) with a large and a narrow initial wave packet in $k$-space, as a function of $\Delta$ and for three different values of $k_{0}$. The black solid lines are for $\sigma=20$, while the dashed red lines are for $\sigma=5$. The considered values of initial momentum are $k_{0}=\frac{\pi}{4}, \frac{\pi}{3}, \frac{\pi}{2}$ for the left, center, and right column, respectively.

In order to compare the error of an estimator with the true value of the parameter to be estimated, we also address the QSNR, defined in Equation (11). Its behavior is shown in the right plot of Figure 3 and in the lower panel of Figure 4, for three different values of the initial central momentum $k_{0}$. The QSNR has a maximum for $\Delta \neq 0$, which corresponds to the value of the barrier height that can be better estimated. As the value of the initial central momentum is increased toward $k_{0}=\frac{\pi}{2}$, the value of the optimal $\Delta$ slightly increases. The dependency on $\sigma$ is negligible when considering the QSNR, as shown in the lower plots, where the behaviors for $\sigma=5$ and $\sigma=20$ are compared. Quite remarkably, the maximum value of the QSNR is very similar, $R_{\mathcal{G}} \approx 1$ for the considered values of $k_{0}$, thus making the initial central momentum a tool to fine tune the optimal value of $\Delta$, but not the corresponding precision.

The behavior of the QSNR has an intuitive and straightforward physical interpretation. If the height of the barrier is negligible $(\Delta \ll 1)$, then the walker is mostly transmitted anyway, and it is very difficult to detect small variations of $\Delta$ itself. Similarly, if $\Delta \gg 1$, the walker is mostly reflected independently of the exact value of $\Delta$. On the other hand, for intermediate values of $\Delta$, the wavefunction of the walker is very sensitive to its value, and measuring the walker indeed provides information. This picture is confirmed if one looks at the zeroth order expression of the QFI in Equation (29), which says that the maxima of the QSNR are located at $\Delta^{2}=2\left[1-\cos \left(2 k_{0}\right)\right]$. Notice that the values of $\left(\Delta, k_{0}\right)$ satisfying this relations are those making the reflection and transmission equal to each other $R\left(\sqrt{2\left[1-\cos \left(2 k_{0}\right)\right]}, k_{0}\right)=$ $T\left(\sqrt{2\left[1-\cos \left(2 k_{0}\right)\right]}, k_{0}\right)=\frac{1}{2}$.

\section{Dichotomic Position Measurement}

We now address the question of whether a realistic position measurement is optimal, i.e., its FI equals the QFI defined in Equation (28). In particular, we consider a dichotomic measurement that just tells us if the particle is located on the left or on the right side of the barrier. Since we know from the Equation (25) 
that the quantities $\rho_{\mathcal{G}}\left(k_{0}, \Delta\right)$ and $\tau_{\mathcal{G}}\left(k_{0}, \Delta\right)$ correspond to the probabilities of finding the particle before or after the obstacle, the FI takes the expression:

$$
\begin{aligned}
F_{\mathcal{G}}\left(k_{0}, \Delta\right) & =\frac{\left[\partial_{\Delta} \rho_{\mathcal{G}}\left(k_{0}, \Delta\right)\right]^{2}}{\rho_{\mathcal{G}}\left(k_{0}, \Delta\right)}+\frac{\left[\partial_{\Delta} \tau_{\mathcal{G}}\left(k_{0}, \Delta\right)\right]^{2}}{\tau_{\mathcal{G}}\left(k_{0}, \Delta\right)}=\frac{\left[\partial_{\Delta} \tau_{\mathcal{G}}\left(k_{0}, \Delta\right)\right]^{2}}{\tau_{\mathcal{G}}\left(k_{0}, \Delta\right)\left[1-\tau_{\mathcal{G}}\left(k_{0}, \Delta\right)\right]} \\
& =\frac{16 \sin ^{2} k_{0}}{\left[2+\Delta^{2}-2 \cos \left(2 k_{0}\right)\right]^{2}}+\frac{g_{F}\left(k_{0}, \Delta\right)}{\sigma^{2}}+O\left(1 / \sigma^{3}\right),
\end{aligned}
$$

where the explicit expression of $g_{F}\left(k_{0}, \Delta\right)$ is reported in Appendix B. As the value of $\sigma$ is increased, i.e., the wave packet is more localized in $k$-space, the FI of the dichotomic measurement approaches the QFI. The second order coefficients $g_{s}\left(k_{0}, \Delta\right), s=H, F$ are different for the QFI and the FI (see Appendix B), but in the range of parameters we explore $\left(\sigma>5,0<\Delta \leq 4,0<k_{0}<\pi\right)$, the ratio $\gamma\left(k_{0}, \Delta\right)=$ $F_{\mathcal{G}}\left(k_{0}, \Delta\right) / H_{\mathcal{G}}\left(k_{0}, \Delta\right)$ is always larger than $\gamma\left(k_{0}, \Delta\right)>0.95$. We conclude that a dichotomic position measurement is nearly optimal to estimate the height of the potential barrier $\Delta$.

\section{Conclusions}

In this work, we introduce and discuss a general probing scheme for scattering problems based on continuous-time quantum walks. In particular, we consider a one-dimensional lattice, with an impurity at its center, i.e., a potential barrier of height $\Delta$, and discuss in details how to quantify the maximum extractable information about the parameter $\Delta$.

Using the continuous-space case as a guide for attacking the problem, we first introduce the single-momentum scattered states $S|k\rangle$ and use them to compute the reflection and transmission coefficients of the considered potential. From the scattered states, we build up the asymptotic Gaussian states, i.e., physical states, that depend, in addition to $\Delta$, on the initial central momentum $k_{0}$ and the spread of the wave packet in position space $\sigma$. We then derive the reflection and transmission probability of such wave packets. Finally, we compute the QFI for the parameter $\Delta$. We show that the QFI has a maximum for $\Delta=0$, and it is strongly affected by the value of $k_{0}$. In particular, values of $k_{0}$ near $\frac{\pi}{2}$ lead to a smaller QFI. Moreover, for $|\Delta| \ll 1$, a small $\sigma$ can increase the precision of the estimation. However, inspection of the QSNR did not show a noticeable difference in its behavior depending on the value of $\sigma$ or $k_{0}$. The QSNR has a maximum for $\Delta \neq 0$, indicating that given the value of the central momentum $k_{0}$, there exists a value for $\Delta$ that can be better estimated, leading to the unit QSNR independently of $\sigma$ and $k_{0}$.

Finally, we investigate the performances of a dichotomic position measurement, which is a binary measurement that is just able to distinguish if a particle is located on the left (reflected) or on the right (transmitted) of the potential barrier. We show that this measurement is optimal, i.e., its FI is equal to the QFI, for large initial wave packets (in position space), while it is nearly optimal for narrow initial wave packets.

Our work paves the way toward the characterization of more involved forms of potentials using a single-particle continuous-time quantum walk as a probe. Extensions of this work may also include more complex structures, such as multi-dimensional graphs, where imperfections created during the fabrication process need to be estimated in order to better control the quantum dynamics over such networks.

Author Contributions: Conceptualization, C.B. and M.G.A.P.; Formal analysis, F.Z., C.B. and M.G.A.P.; Investigation, F.Z.; Methodology, C.B. and M.G.A.P.; Software, F.Z.; Supervision, M.G.A.P.; Validation, C.B. and M.G.A.P.; Visualization, C.B.; Writing—original draft, F.Z. and C.B.; Writing—review \& editing, C.B. and M.G.A.P. All authors have read and agreed to the published version of the manuscript.

Funding: This research received no external funding.

Acknowledgments: MGAP is a member of INdAM-GNFM. 
Conflicts of Interest: The authors declare no conflict of interest.

\section{Abbreviations}

The following abbreviations are used in this manuscript:

CTQW Continuous-time quantum walk

CR Cramér-Rao

FI Fisher information

QFI Quantum Fisher information

QSNR Quantum signal-to-noise ratio

\section{Appendix A. Gaussian Wave Packet in K-Space}

Consider the Gaussian wave packet in position space defined by Equation (21). Here, we show that its expression in $k$-space, within certain approximations, is given by Expression (23). We start by considering the the projection of Equation (21) into a state $|k\rangle$ :

$$
\left\langle k \mid \mathcal{G}_{k_{0}}\right\rangle=\frac{\mathcal{N}}{\sqrt{2 \pi}} \sum_{j \in \mathbb{Z}} e^{-\frac{(j-\mu)^{2}}{2 \sigma^{2}}} e^{i\left(k_{0}-k\right) j}
$$

The infinite sum can be calculated using the Poisson summation formula, which states that, for suitable functions $f: \sum_{j \in \mathbb{Z}} f(j)=\sum_{n \in \mathbb{Z}} \hat{f}(n)=\sum_{n \in \mathbb{Z}} \int_{-\infty}^{+\infty} f(x) e^{-i 2 \pi n x} \mathrm{~d} x$. In our particular case:

$$
\left\langle k \mid \mathcal{G}_{k_{0}}\right\rangle=\frac{\mathcal{N}}{\sqrt{2 \pi}} \sum_{j \in \mathbb{Z}} e^{-\frac{(j-\mu)^{2}}{2 \sigma^{2}}} e^{i\left(k_{0}-k\right) j}=\frac{\mathcal{N}}{\sqrt{2 \pi}} \sum_{n \in \mathbb{Z}} \int_{-\infty}^{+\infty} e^{-\frac{(x-\mu)^{2}}{2 \sigma^{2}}} e^{i\left(k_{0}-k\right) x} e^{-i 2 \pi n x} \mathrm{~d} x .
$$

The last integral is a continuous Fourier transform of a Gaussian function; therefore:

$$
\int_{-\infty}^{+\infty} e^{-\frac{(x-\mu)^{2}}{2 \sigma^{2}}} e^{i\left(k_{0}-k\right) x} e^{-i 2 \pi n x} \mathrm{~d} x=\sqrt{2 \pi \sigma^{2}} e^{-\frac{\left(2 \pi n+k-k_{0}\right)^{2}}{2 \frac{1}{\sigma^{2}}}} e^{-i \mu\left(2 \pi n+k-k_{0}\right) .}
$$

Inserting Equation (A3) into (A2) (discarding the constant global phase $e^{i \mu k_{0}}$ ), we obtain:

$$
\left\langle k \mid \mathcal{G}_{k_{0}}\right\rangle=\mathcal{N} \sigma \sum_{n \in \mathbb{Z}} e^{-\frac{\left(2 \pi n+k-k_{0}\right)^{2}}{2 \frac{1}{\sigma^{2}}}} e^{-i \mu(2 \pi n+k)} .
$$

The transformed state is not a Gaussian state, but it is an infinite sum of Gaussian states periodically displaced. However, if the wave packet is localized enough in reciprocal space, it is possible to approximate the last infinite summation by keeping only the central term $n=0$ (it is always possible to shift the definition of $k$ and $k_{0}$ in the interval $[-\pi, \pi)$ because they are defined modulo $2 \pi$ ). The localization assumption is needed in order to consider only one term, otherwise the tails of adjacent Gaussian functions could overlap. In other words, $k_{0}$ should be far away from the boundaries of the interval $[-\pi, \pi)$, i.e., $\left|k_{0}-\pi\right|$ and $\left|k_{0}+\pi\right|$ should be much larger than the standard deviation in the reciprocal space. Overall, the conditions read as $\min \left(\left|k_{0}-\pi\right|,\left|k_{0}+\pi\right|\right) \gg 1 / \sigma$. With this assumption:

$$
\mathcal{G}_{k_{0}}(k)=\left\langle k \mid \mathcal{G}_{k_{0}}\right\rangle \approx \mathcal{N} \sigma e^{-\frac{\left(k-k_{0}\right)^{2}}{2 \frac{1}{\sigma^{2}}}} e^{-i \mu k} .
$$


Thus, a discrete Gaussian state in the position basis remains a Gaussian state in reciprocal space within the considered approximation. The calculation of the normalization constant $\mathcal{N}$ reduces to the calculation of a Gaussian integral:

$$
1=\int_{-\pi}^{\pi}\left|\mathcal{G}_{k_{0}}(k)\right|^{2} \mathrm{~d} k \approx \int_{-\infty}^{\infty}\left|\mathcal{G}_{k_{0}}(k)\right|^{2} \mathrm{~d} k=|\mathcal{N}|^{2} \sigma \sqrt{\pi}
$$

with:

$$
|\mathcal{N}|^{2} \approx \frac{1}{\sqrt{\pi \sigma^{2}}}
$$

\section{Appendix B. The Explicit Expression of the Functions $g_{H}\left(\Delta, k_{0}\right)$ and $g_{F}\left(\Delta, k_{0}\right)$}

We have:

$$
\begin{aligned}
g_{H}\left(k_{0}, \Delta\right) & =\frac{4\left[3 \cos 6 k_{0}+2\left(5 \Delta^{2}-1\right) \cos 4 k_{0}+3\left(3 \Delta^{4}-19\right) \cos 2 k_{0}+\Delta^{4}-10 \Delta^{2}+18\right]}{\left[\Delta^{2}+2\left(1-\cos 2 k_{0}\right)\right]^{4}} \\
g_{F}\left(k_{0}, \Delta\right) & =\frac{8\left[\cos 6 k_{0}+6 \Delta^{2} \cos 4 k_{0}+\left(\Delta^{4}-9\right) \cos 2 k_{0}-6 \Delta^{2}+8\right]}{\left[\Delta^{2}+2\left(1-\cos 2 k_{0}\right)\right]^{4}}
\end{aligned}
$$

\section{References}

1. Rutherford, E.F. LXXIX. The scattering of $\alpha$ and $\beta$ particles by matter and the structure of the atom. Philos. Mag. Ser. 1911, 21, 669-688. [CrossRef]

2. Franklin, R. Influence of the Bonding Electrons on the Scattering of X-Rays by Carbon. Nature 1950, 165, 71-72. [CrossRef] [PubMed]

3. Chamberlain, O.; Segrè, E.; Wiegand, C.; Ypsilantis, T. Observation of Antiprotons. Phys. Rev. 1955, 100, 947-950. [CrossRef]

4. Aad, G.Et Al.. Obs. A New Part. Search Stand. Model Higgs Boson ATLAS Detect. LHC. Phys. Lett. B 2012, 716, 1-29. [CrossRef]

5. Helstrom, C.W. Quantum Detection and Estimation Theory; Academic Press: New York, NY, USA, 1976.

6. Gebbia, F.; Benedetti, C.; Benatti, F.; Floreanini, R.; Bina, M.; Paris, M.G.A. Two-qubit quantum probes for the temperature of an Ohmic environment. Phys. Rev. A 2020, 101, 032112. [CrossRef]

7. Tamascelli, D.; Benedetti, C.; Breuer, H.P.; Paris, M. Quantum probing beyond pure dephasing. New J. Phys. 2020, 22, 083027. [CrossRef]

8. Salari Sehdaran, F.; Bina, M.; Benedetti, C.; Paris, M. Quantum Probes for Ohmic Environments at Thermal Equilibrium. Entropy 2019, 21, 486. [CrossRef]

9. Mirkin, N.; Larocca, M.; Wisniacki, D. Quantum metrology in a non-Markovian quantum evolution. Phys. Rev. A 2020, 102, 022618. [CrossRef]

10. Wu, W.; Shi, C. Quantum parameter estimation in a dissipative environment. Phys. Rev. A 2020, 102, 032607. [CrossRef]

11. Seveso, L.; Benedetti, C.; Paris, M. The walker speaks its graph: Global and nearly-local probing of the tunnelling amplitude in continuous-time quantum walks. J. Phys. A Math. Theor. 2019, 52, 105304. [CrossRef]

12. Tamascelli, D.; Benedetti, C.; Olivares, S.; Paris, M.G.A. Characterization of qubit chains by Feynman probes. Phys. Rev. A 2016, 94, 042129. [CrossRef]

13. Razzoli, L.; Ghirardi, L.; Siloi, I.; Bordone, P.; Paris, M.G.A. Lattice quantum magnetometry. Phys. Rev. A 2019, 99, 062330. [CrossRef] 
14. Benedetti, C.; Paris, M. Characterization of classical Gaussian processes using quantum probes. Phys. Lett. A 2014, 378, 2495-2500. [CrossRef]

15. Schreiber, A.; Cassemiro, K.N.; Potoček, V.; Gábris, A.; Jex, I.; Silberhorn, C. Decoherence and Disorder in Quantum Walks: From Ballistic Spread to Localization. Phys. Rev. Lett. 2011, 106, 180403. [CrossRef]

16. Izaac, J.A.; Wang, J.B.; Li, Z.J. Continuous-time quantum walks with defects and disorder. Phys. Rev. A 2013, 88, 042334. [CrossRef]

17. Li, Z.J.; Izaac, J.A.; Wang, J.B. Position-defect-induced reflection, trapping, transmission, and resonance in quantum walks. Phys. Rev. A 2013, 87, 012314. [CrossRef]

18. Li, Z.; Wang, J. Single-point position and transition defects in continuous time quantum walks. Sci. Rep. 2015, 5, 13585. [CrossRef]

19. de Falco, D.; Tamascelli, D. Noise-assisted quantum transport and computation. J. Phys. A Math. Theor. 2013, 46, 225301. [CrossRef]

20. Chakraborty, S.; Novo, L.; Di Giorgio, S.; Omar, Y. Optimal Quantum Spatial Search on Random Temporal Networks. Phys. Rev. Lett. 2017, 119, 220503. [CrossRef]

21. Cattaneo, M.; Rossi, M.A.C.; Paris, M.G.A.; Maniscalco, S. Quantum spatial search on graphs subject to dynamical noise. Phys. Rev. A 2018, 98, 052347. [CrossRef]

22. Morley, J.G.; Chancellor, N.; Bose, S.; Kendon, V. Quantum search with hybrid adiabatic-quantum-walk algorithms and realistic noise. Phys. Rev. A 2019, 99, 022339. [CrossRef]

23. Benedetti, C.; Rossi, M.A.C.; Paris, M.G.A. Continuous-time quantum walks on dynamical percolation graphs. EPL 2019, 124, 60001. [CrossRef]

24. Boykin, T.B.; Klimeck, G. The discretized Schrödinger equation and simple models for semiconductor quantum wells. Eur. J. Phys. 2004, 25, 503-514. [CrossRef]

25. Tarasov, V.E. Exact discretization of Schrödinger equation. Phys. Lett. A 2016, 380, 68-75. [CrossRef]

26. Farhi, E.; Gutmann, S. Quantum computation and decision trees. Phys. Rev. A 1998, 58, 915-928. [CrossRef]

27. Mülken, O.; Blumen, A. Continuous-time quantum walks: Models for coherent transport on complex networks. Phys. Rep. 2011, 502, 37-87. [CrossRef]

28. Wong, T.G.; Tarrataca, L.; Nahimov, N. Laplacian versus adjacency matrix in quantum walk search. Quantum Inf. Proc. 2016, 15, 4029-4048. [CrossRef]

29. Simon, S.H. The Oxford Solid State Basics; The Oxford University Press: Oxford,UK, 2013.

30. Paris, M.G.A. Quantum estimation for quantum technology. Int. J. Quantum Inf. 2009, 7, 125-137. [CrossRef]

31. Griffiths, D.J. Introduction to Quantum Mechanics, 2nd ed.; Prentice Hall: Upper Saddle River, NJ, USA, 2005.

Publisher's Note: MDPI stays neutral with regard to jurisdictional claims in published maps and institutional affiliations. 